\title{
Research on High-Quality Development of Auto Parts Manufacturing Industry Based on Machine Learning Model
}

\author{
Xuchang Yang, Qian Zheng, Yueying Hu, Ronghui Chen $(\mathbb{D}$, Xuepeng Wang, and Yanan Liu
}

College of Management, Anhui Science and Technology University, Bengbu 233030, China

Correspondence should be addressed to Ronghui Chen; chenrh@ahstu.edu.cn

Received 29 December 2021; Accepted 17 January 2022; Published 10 February 2022

Academic Editor: Man Fai Leung

Copyright ( 92022 Xuchang Yang et al. This is an open access article distributed under the Creative Commons Attribution License, which permits unrestricted use, distribution, and reproduction in any medium, provided the original work is properly cited.

\begin{abstract}
At present, in China's automobile manufacturing industry, the main problem is the manufacturing of parts and on-board equipment. Most domestic industries still adopt the step-by-step production of parts, and each manufacturer customizes the required parts according to the scale and production needs of its own enterprise. This situation is easy to cause unstable quality of parts and serious unqualified quality inspection problems. Based on the above situation, we study the high-quality development, parts quality optimization, and remanufacturing of auto parts manufacturing industry with the support of machine learning model. Firstly, based on the analysis of auto parts procurement and production mode, this paper briefly describes the basic problems in the manufacturing process of auto parts in China. Machine learning technology is used to count the changes of quality data in manufacturing, and the quality standard is reflected in the learning model. The machine learning algorithm is used to diagnose and analyze the faults of auto parts and equipment, so as to turn high-quality production to high-quality production. The projection feature extraction algorithm is used to quantitatively analyze the low quality state of automobile parts. Finally, 3D printing technology is used to solve the quality manufacturing problem of parts with high-precision requirements, and the later materials are processed again to achieve the purpose of remanufacturing planning. The results show that the transformation of auto parts manufacturing to high quality can improve the economic development of the auto industry and meet the needs of modern society. The data analysis of parts controlled by machine learning model can help the precision manufacturing of automobile parts.
\end{abstract}

\section{Introduction}

With the rapid development of China's economic strength, the parts manufacturing industry in the automotive industry has also started a wide range of production and processing (Chen Min et al.) [1]. The advantages of auto parts development can promote the transformation and innovation of the auto industry. The parts manufacturing industry needs to focus on high-tech quality transformation and strictly control the quality of its products to meet the needs of modern cities. In different regions of China, the development of auto parts manufacturing industry is different, and most cities with high-tech technology are relatively smooth (Chen et al.) [2]. Auto parts manufacturing needs to be supported by the urban economy and obtain new transformation opportunities from the development of high-tech technology. Solving the problem of auto parts manufacturing can improve the economic benefits of the whole city, which plays an important role and helps in urban innovation and development ( $\mathrm{Wu}$ et al.) [3]. At present, the economic benefits that China's auto parts manufacturing industry can create are limited by many reasons. Enterprises can not control the overall quality of parts in normal operation. The sales scope and export channels of auto parts are relatively few, and cross international exchanges and transactions cannot be realized globally (Lu Yanqing et al. 2021) [4]. The above factors are unfavorable to the development of China's automobile industry, especially the quality problems caused by auto parts, which has always been the primary link to be solved (Jin Hongji et al. 2021) [5]. Our government has promulgated many new policies according to the development 
status of the automobile industry, and there are new standards and restrictions in quality control and enterprise management. With the advent of the Internet and big data environment, new technology development can help optimize and reform the quality of auto parts manufacturing industry (Kong Ningning et al.) [6].

The main factors affecting the quality change of China's auto parts manufacturing industry also include production cost. Each enterprise has its own cost range, and the reduction of profits leads to many problems in enterprise production ( $\mathrm{Hu}$ Chun et al. 2021) [7]. The investment growth of foreign investors in the industry is low, and this bad cycle leads to the failure of new technologies in time. The traditional processing method of auto parts is relatively single, and the product renewal and creativity have not been optimized and improved (Qiu Shaohu et al. 2021) [8]. Obviously, the processing efficiency can not meet the production demand of the current automobile industry. At present, the following problems are easy to appear in the process of auto parts manufacturing: first, the production line in many manufacturing industries is too long, which makes it easy to slow down the progress and efficiency of engineering manufacturing in complex environment. The enterprise does not control and allocate the production management, and the complexity of the production line has brought many work difficulties to most employees (Jinxi et al. 2021) [9]. The problems in the production process can not be found immediately. In order to improve the quality of parts, it needs to be analyzed from the production process. Finally, the development of China's automobile industry is relatively diversified and there are many kinds of automobiles. This diversity leads to the need to consider processing parts from many aspects. Therefore, in the development of parts manufacturing industry, it is necessary to increase the types and characteristics of products, improve their own quality, and stand out from various industry competitions (Wang Chuan et al. 2021) [10].

This paper puts forward new measures and ideas for the reuse of parts in China. Its innovative contributions are as follows: 1. Starting from the market economy of automobile products, this paper discusses the influence of cost price and material selection on automobile performance. Starting with the quality control management of parts, the core structure in the manufacturing process is improved. 2. Introduce machine learning model to statistically analyze material data. The training samples are used to obtain the optimal results of quality data, which are added to the manufacturing process of auto parts. 3. Based on the actual situation of China's automobile industry, the fault data are extracted from the part processing process, and the quality parameters are optimized by using the simulation environment. In order to improve the manufacturing quality of parts, machine learning model is used to train the fault analysis performance in sample making.

This paper is mainly divided into three parts. The first part mainly describes the background of automobile industry and automobile parts manufacturing industry and analyzes the current situation of the application of machine learning algorithm in various industries from the perspective of parts manufacturing process. The second part first analyzes the quality control problems in the process of auto parts manufacturing and transforms the trend of parts manufacturing into high-quality development. Starting with the control of raw materials, the influencing factors of material selection on quality are analyzed by using big data and machine learning algorithm. After data acquisition, the monitoring effect of machine learning model on part manufacturing is analyzed. $3 \mathrm{D}$ printing technology is adopted to increase material selectivity and optimize and improve individual parts with high-precision requirements. Finally, it analyzes the industry discovery trend and optimizes the quality problems from the concept of parts remanufacturing in the automotive industry. The third part analyzes the results of high-quality transformation of auto parts manufacturing industry based on machine learning model, as well as the research on parts remanufacturing and quality optimization.

\section{Related Work}

In the process of automobile manufacturing, it is necessary to analyze and investigate the quality and process of parts. The selection of raw material quality of parts and components has a great impact on the results of the whole manufacturing (Zhang Shibin et al.) [11]. Therefore, it is necessary to collect and analyze the data before the use of raw materials. The statistical results of data analysis are constructed by using machine learning model. Machine learning model can be widely used in computer field, natural language field, and intelligent recognition field (Liu Qixu et al. 2021) [12]. It provides accurate data support and algorithm support for the above industries. Most machine learning models can show good results in sample data detection and prediction. However, the training data of the model needs to select the appropriate range and input method. In order to improve the quality development in automobile parts manufacturing, we need to build a relatively safe and reliable machine learning model. The risk analysis and data prediction of learning model are used to ensure the quality standard of parts. In the training of machine learning model, it is also necessary to ensure the integrity of data and detect the location and specific factors of automobile component faults according to the above data (Wang et al. 2021) [13].

Water conservancy projects in Japan have always been the main industry with risks and accidents. In order to enhance the accuracy of water conservancy work management and risk assessment, they added machine learning algorithm to Engineering Research (Liu Haipeng et al. 2021) [14]. Using machine learning model to construct data association structure, risk assessment and data prediction are carried out for the operation links of water conservancy projects. Under the influence of human beings, the mining effect in the data framework of hydraulic engineering can analyze the key influencing factors and establish corresponding emergency treatment and guarantee measures. With the support of computer technology, many intelligent robots are also widely used in people's life. It is easy for 
intelligent robot to face the danger of obstacles in the process of moving. In order to improve the robot's ability to avoid obstacles in real time, it is necessary to use machine learning algorithm to transform the robot system (Yu Hong et al. 2021) [15]. Machine learning algorithm is used to judge the distance of obstacles in real time, and the travel direction is planned. The results show that the robot optimized by the algorithm can form a reliable and stable path in motion. The development of China's automotive machinery field is a hot industry in the near future. Automotive machinery and equipment are prone to many problems in operation. In order to solve the fault problem and improve the diagnosis accuracy, we use machine learning algorithm to ensure the normal operation of automobile mechanical equipment. With its own advantages, machine learning algorithm can change the structure of data calculation and realize fault diagnosis and real-time monitoring of mechanical equipment. Based on the application status of machine learning algorithm in various fields, this paper studies the highquality transformation and quality optimization measures of auto parts manufacturing industry.

\section{Methodology}

3.1. Research on High-Quality Transformation of Auto Parts Manufacturing Based on Machine Learning Model. With the continuous improvement of China's economic development, people's use frequency and ownership penetration rate of cars are increasing exponentially. With the growth of automobile sales, the development of the industry is also facing a new turn. The demand for the quality of auto parts and equipment in the auto market is higher and higher. When choosing an automobile brand, we first need to consider its quality and durability. The quality standard of auto parts also provides a data reference for selecting auto brands. Steadily promoting the development of China's automobile industry can play a positive role in national economic growth. At present, although China's automobile has basically reached the state of popularization, people's demand for high quality is becoming closer and closer. The shift of auto parts from quantitative development to highquality development has become the main factor affecting the process of the industry. From the analysis of automobile sales market, we try to reduce the gap with partners in terms of quality requirements and shorten the distance from manufacturing process and core technology, control automotive equipment and related parts, and constantly innovate and optimize to achieve the purpose of stable industrial development.

Most manufacturers in China's automobile manufacturing industry contract to the corresponding units for parts manufacturing, which is unfavorable to the control of parts quality. It is easy to cause unqualified parts, which can directly affect the overall quality of automotive products. The production process is mainly composed of several processes, as shown in Figure 1.

As can be seen from Figure 1, firstly, it is divided in part manufacturing for different automobile types. Product quality control is divided into inspection stage, statistical data stage, data detection stage, and overall quality management stage. According to the characteristics of auto parts and the operation process, select the appropriate detection method. Manually control the composition of parts in quality inspection. According to the above process, the supervision of parts and automobile quality can be realized. Although the quality problems can be mastered in their own production links, there are also prominent defects. For example, the order of parts in production will bring some difficulties to quality monitoring, which not only reduces the production efficiency, but also expands the actual cost. In order to improve the overall quality of parts, the industry can only transform active intervention into passive intervention and control. At this stage, parts production is still the focus of research. Many data will be used in the process of component quality accounting, and the work content of this large amount of data calculation is more complex. The use of raw material parameters and the accuracy of calculation data will bring some difficulties to quality accounting. In order to solve the problem of data accounting, this paper uses machine learning algorithm to construct data analysis model.

Machine learning algorithm can select targeted detection methods according to the detection requirements in automobile parts manufacturing. Carry out data detection and tracking from raw material distribution and manufacturing process. The test data in the process of parts manufacturing is divided into two parts. One is the data information inside the part, and the other is the detection information of external equipment. In order to better realize tracking and analysis in component production, we need machine learning algorithm as technical support. A mathematical model is established for the internal and external inspection data of parts, and the position of inspection nodes is defined by data analysis technology. Firstly, the parts manufacturing of an automobile product is randomly selected as the prototype. The internal inspection data needs to correct the position of the inspection node. We define the gap between the front and rear positions of the main target as follows:

$$
\arg \min L[g(H), Z] .
$$

In the formula, $H$ is the actual position of the detection node and $Z$ is the corresponding position of the specific data. The capture function in machine learning algorithm is used to define the relationship of data quality inspection gap. The input and output variable formula of the model is

$$
\begin{aligned}
X_{n \times m} & =\left(\begin{array}{c}
x_{1} x_{2} \ldots x_{m} \\
x_{2} x_{2} \ldots x_{n} \\
x_{n} x_{n} \ldots x_{n m}
\end{array}\right), \\
Y_{n} & =\left(\begin{array}{c}
y_{1} \\
y_{2} \\
\ldots \\
y_{n}
\end{array}\right) .
\end{aligned}
$$

In the formula, $n$ variable is the number of samples and $m$ is the number of eigenvalues of the input data. The mapping relationship between data nodes in machine 


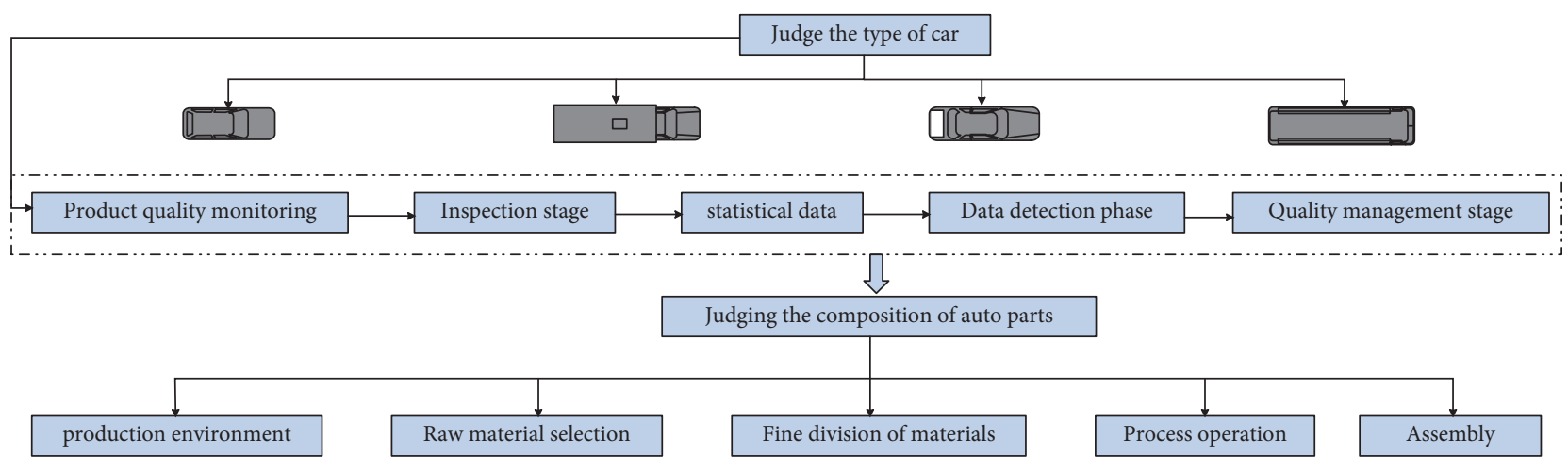

Figure 1: Production process.

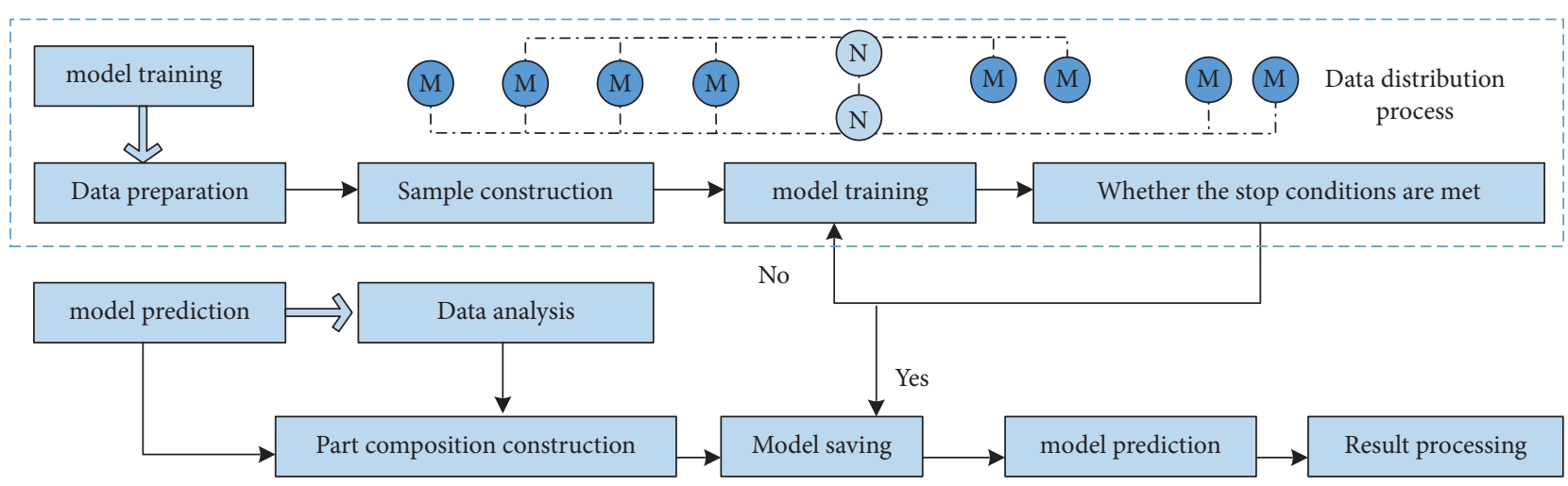

FIgURE 2: Quality data enhancement process constructed by machine learning algorithm.

learning model is reflected by linear regression analysis. It can also quickly judge the correlation between data input and output in feature screening. The calculation formula is

$$
f(X)=W_{T} X+B .
$$

A large amount of data needs to be added to the machine learning model for training. Select the data with stable iteration times to obtain accurate data results. The ratio between the predicted value of error measurement and the actual data is used to optimize the parameter model. The error coefficient can be obtained according to the following formula:

$$
E_{d}=\frac{1}{n} \sum_{l=1}^{n}\left(\widehat{y}_{t}-y_{t}\right) .
$$

$y_{t}$ and $\bar{y}_{t}$ represent the actual value and the predicted value, respectively. In the data mining in Figure 2, it is necessary to judge the location relationship of data nodes. The relationship between internal and external positions of parts is predicted and analyzed, and the purpose of data enhancement is constructed through linear regression. The quality data enhancement process constructed by machine learning algorithm is shown in Figure 2.

As can be seen from Figure 2, the data generated in the manufacturing of parts are obtained. Build corresponding models for different vehicle samples, preprocess the mapping data, and generate sample cases. In the training model, an appropriate algorithm is used to optimize the quality parameters. Save the optimal parameter model for result processing. Test the influence range of different data on the quality of parts. Due to the technical differences between different manufacturers, dynamic data changes will occur in each component manufacturing engineering. The best quality data we calculated can only provide a certain range of reference efficacy. Next, we need to further study the quality optimization and specific measures of auto parts.

\subsection{Research on Remanufacturing and Quality Optimization} of Auto Parts Based on Machine Learning Model. The manufacturing quality of automobile parts plays an important role and influence on the overall performance and sales of automobile. Parts manufacturing cost and core technology have a great relationship with quality optimization. In quality control, the selection and processing of raw materials can directly affect the change of quality coefficient in production. Raw materials and customer quality cost are internal quality problems of parts. In order to improve the overall performance of automobile, we also need to explore the factors affecting quality from actual production and manufacturing. Step errors or operation failures in the manufacturing process will affect the quality parameters of parts. We also need to detect and judge all aspects of parts in automobile quality inspection. In order to improve the accuracy and effectiveness of fault detection, a component model based on machine learning algorithm needs to be added to the manufacturing process. Control the production 
process through the characteristic changes in the manufacturing process of parts. Judge whether there is a manufacturing fault according to the characteristic data. If there is a fault, it can be handled and located in time. Firstly, the projection algorithm is used to extract the fault features in the manufacturing process of auto parts. The model is represented by spatial matrix coefficients:

$$
\min \sum\|L\|,+\lambda E_{2.1}, s, 1, X=X L
$$

In the formula, the variable $\lambda E_{2.1}$ represents the fault characteristic data. We judge whether there are similar variables from the matrix data and simulate the information of the same sample:

$$
\begin{aligned}
x_{i j} & =X^{w} i j+E_{i j} \\
& \left.=\sum_{i=1}^{c} X w_{i}^{s} j\right)+e_{i j},
\end{aligned}
$$

where $X$ represents the noise data generated by the fault. The approximate position change of fault is simulated by sample similarity vector. If the training sample does not change, then

$$
y=P^{T} .
$$

The calculation variation of the above formula is

$$
\begin{aligned}
y_{i j}= & p\left(X w_{i j}+e\right) \\
= & \sum_{x-1}^{c} p^{T} X_{W} i j+P^{T} e_{i j}, \\
& P^{T} X_{i} w_{i j}^{i}+\sum_{x=1}^{c} P^{T} X w_{s}^{i j}+p^{t} e_{i j} .
\end{aligned}
$$

In order to improve the accuracy of the analysis data in the calculation, we need to control the measurement within the minimum distance. The minimum distance is defined as

$$
\left\|y_{i j}-p^{T} X w_{i j}^{s}\right\|^{2} \text {. }
$$

Therefore, in the manufacturing process, the specific problem can be analyzed through the fault space. The transformation calculation formula is

$$
\sum_{n}^{i j} \sum_{m}^{i j}\left\|y_{i j}-p^{T} X w_{i j}^{s}\right\|^{2}=\operatorname{tr}\left(p^{T} S_{b}^{L} p\right) .
$$

In the formula, in order to achieve reasonable data distribution and keep the accuracy above the standard coefficient, the minimum norm needs to be planned:

$$
\begin{aligned}
& \sum_{i j}\left\|P_{e i j}^{T}\right\|=\sum_{i j}\left\|p^{t}\left(x_{i j}-x_{s} w_{i j}^{s}\right)\right\|^{2}, \\
& \sum_{i j}\left\|P_{e i j}^{T}\right\|=\operatorname{tr}\left(p_{s}^{T} S_{e}^{L} p\right) .
\end{aligned}
$$

In order to make the calculation process simpler, we can also use the identity matrix to express it. The mean value of the above results is the highest, and the vector of the projection matrix can be represented by the generalized eigenvalue. Finally, in the data analysis level, the part of identifying fault characteristics needs to be judged by data units. The machine learning model is highly adaptable in automatic learning. We use clustering conditions to plan the automatic learning efficiency of machine learning:

$$
\eta(t+q)=\eta_{0}\left(\frac{a_{1}}{\eta_{0}}\right)^{s} .
$$

According to the above formula and machine learning model, the quality changes of parts can be monitored in real time in the manufacturing process, and the steps beyond the stable range can be rearranged and combined. In order to further reflect the effect of parts quality optimization, we can also start from parts remanufacturing. Conduct secondary processing and treatment for unqualified parts and improve the quality coefficient to the standard range and flow into the market again. We investigated the sales of remanufactured products of automobiles and parts, as shown in Figure 3.

It can be seen from Figure 3 that, with the increase of years, the transaction amount in China's automobile market has exceeded the upper limit over the years. Parts remanufacturing accounts for a large proportion in the market. It shows that good sales results can be achieved after secondary processing and improving quality standards. In the quality optimization measures, machine learning model is used to monitor the manufacturing process, which brings core technical support to the secondary manufacturing of auto parts.

\section{Result Analysis and Discussion}

4.1. Analysis of Research Results of High-Quality Transformation of Automobile Parts Manufacturing Based on Machine Learning Model. In the above research, we found that controlling the quality coefficient of automobile parts can improve the overall performance of automobile. Quality inspection of selected materials should be carried out in the manufacturing process of auto parts. After data acquisition, simulation comparison is carried out to analyze the influence range of material parameters on parts. In order to save costs and increase competitive advantage, many manufacturers will choose to reduce raw material indicators, which affects the purpose of the transformation of the auto parts industry to high quality to a certain extent. This paper establishes a machine learning model to study the high-quality transformation of parts. Firstly, a certain automobile product is randomly selected to establish a virtual model for the distribution of parts and components, as shown in Figure 4.

It can be seen from Figure 4 that the distribution of parts in the automobile structure is complex, and there are many factors affecting the overall quality. We need to fit the overall data and conduct unified calculation in the manufacturing and inspection of parts. In the detection, the change of actual quality data is significantly greater than that of predicted data, so it needs to be subdivided by manual intervention. Estimate the key positions in parts manufacturing from 


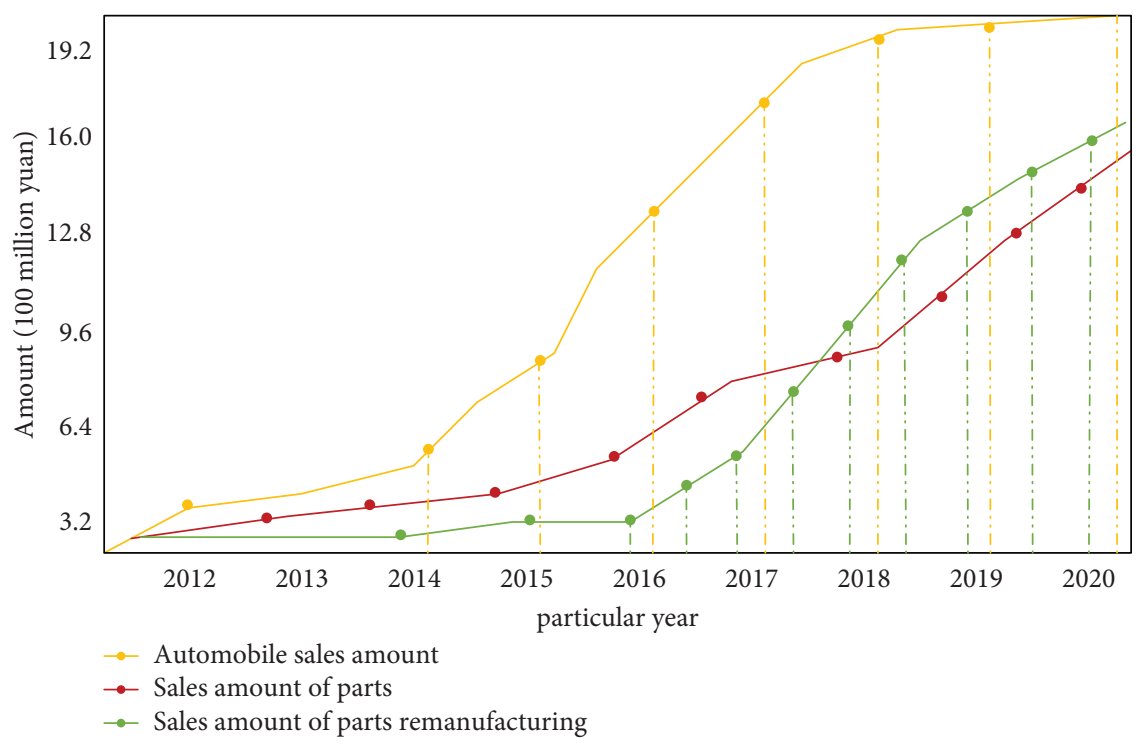

Figure 3: Sales of remanufactured products of automobiles and parts.

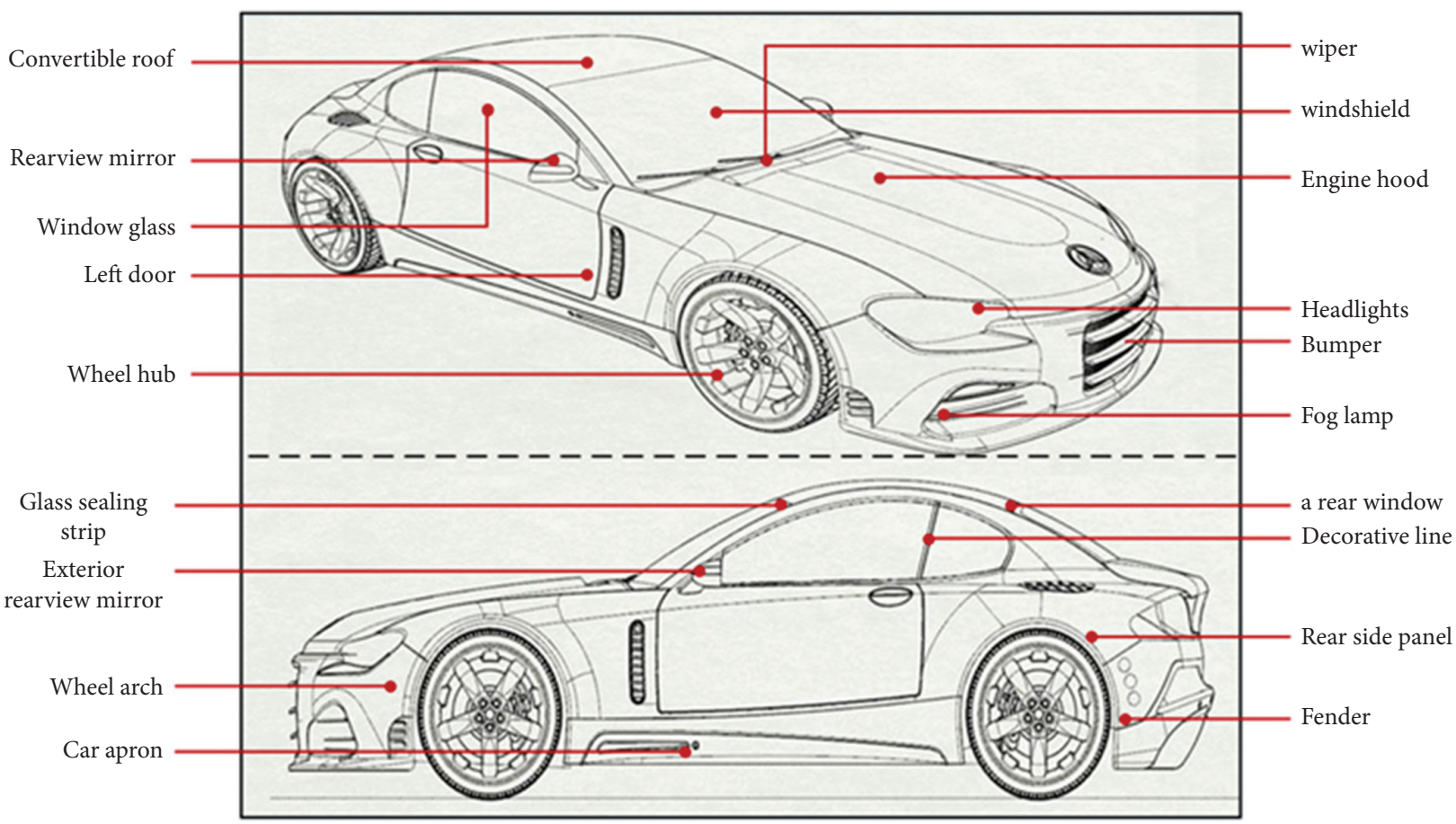

Figure 4: Virtual model of automobile parts distribution.

different angles, and record the position changes and material selection. We compare the quality parameters of parts processed with different raw materials, as shown in Figure 5.

As can be seen from Figure 5, with the gradual increase of the stiffness of raw materials, the quality parameters of corresponding products also increase. The material data predicted by machine learning model can highly meet the product demand. Therefore, this algorithm has obvious advantages in practical application. Finally, we can also use $3 \mathrm{D}$ printing to solve the problem of high-precision design parts.
The higher the precision, the higher the quality of the finished product delivered by printing. Therefore, for highprecision $3 \mathrm{D}$ printing, the primary technical difficulty is the printing precision, that is, the optical resolution: the size of a single pixel of the projection light. Printing speed is also one of the technical difficulties to be broken through in high-precision 3D printing. The formation process of $P \mu$ SL 3D printing technology is as follows: firstly, the three-dimensional structure model is constructed by modeling software; then, the slicing software is used to slice the three-dimensional model with a certain layer thickness to obtain a series of two-dimensional 


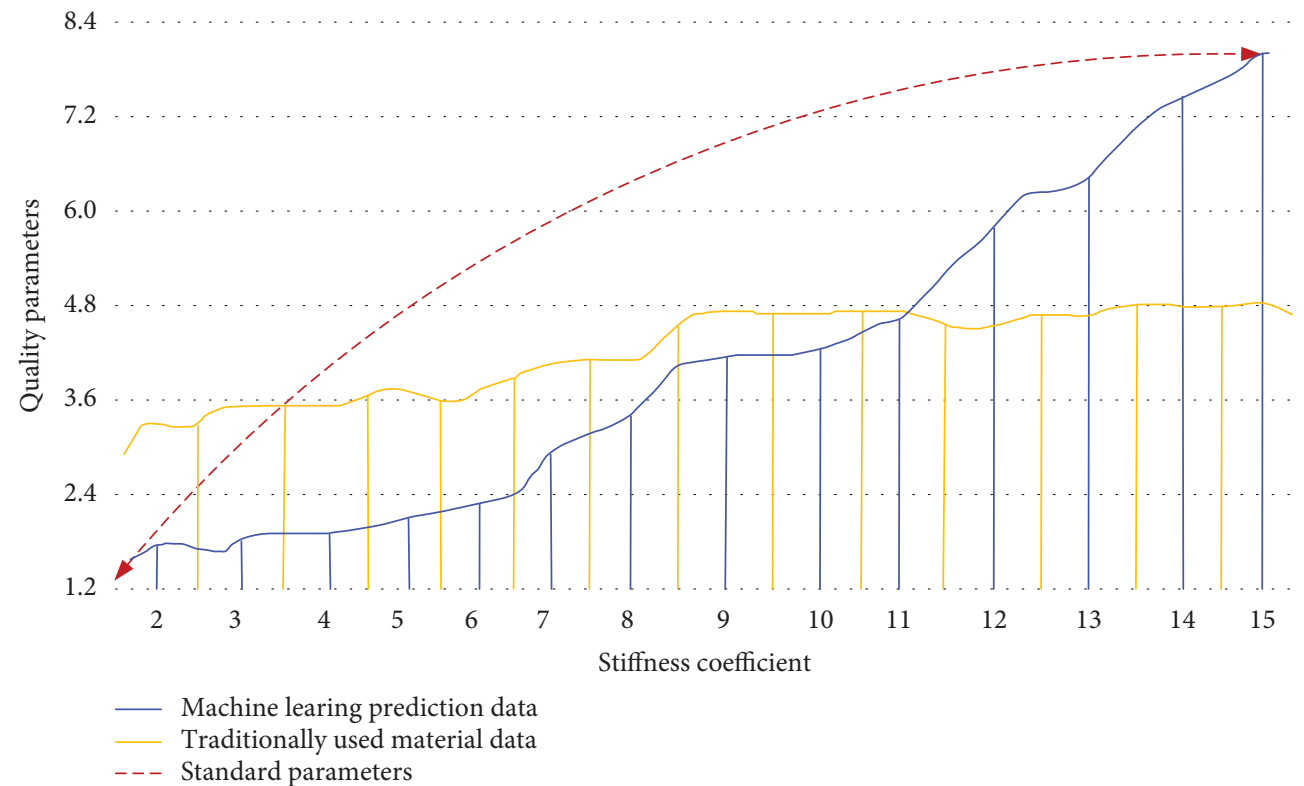

FIGURE 5: Comparison of quality parameters of parts processed with different raw materials.

pictures with specific patterns; then use P $\mu$ SL 3D printing system performs full face projection exposure on each layer of pattern after slicing. Repeat the previous step repeatedly and stack layers to finally form the required three-dimensional structure. In addition, the printing system can also splice and print large-size samples through the movement of the printing platform, so as to realize high-precision, large format, and cross-scale processing.

The corresponding shapes and structures are generated directly from the virtual platform. Using 3D printing technology can also reduce the cost of parts and improve the precision of products. Among them, this technology can be used in shape design, interior decoration matching, plug-ins, and other links.

4.2. Analysis of Research Results of Automobile Parts Remanufacturing and Quality Optimization Based on Machine Learning Model. Improving the quality problems in automobile parts manufacturing needs to be considered from many aspects. Firstly, to improve the quality management of parts, it is necessary to establish the corresponding management model and standard rules. This paper analyzes the characteristics of China's automobile industry and carries out targeted transformation from the domestic environment. Introduce reasonable control measures into quality control and management and establish corresponding departments. Take quality optimization as the core part of manufacturing process. Secondly, in the process of manufacturing, it is also necessary to ensure the transparency of parts production and ensure that each link can be monitored in real time. Record the problems encountered in the production process and achieve accurate positioning. The automatic training model is established according to the recorded information of fault location, and the automatic learning model is transformed and upgraded by machine learning algorithm. That is, in the face of the next quality problem, the fault node can be located in time to achieve targeted solution. Firstly, we analyze the problem of low quality caused by incorrect operation in automobile parts manufacturing, select a certain position of automobile parts to establish a fault analysis model, and use 3D printing technology to compare the internal structure of normal parts and faulty parts, as shown in Figure 6.

We extract the feature points of the model from the automobile parts structure in Figure 6 and verify the accuracy of the data through comparative experiments. At the same time, it is added to the machine learning training model to synchronize multiple fault factors for common analysis. The change trend of component quality parameters after training with machine learning model is shown in Figure 7.

It can be seen from Figure 7 that, with the enhancement of model training time, the quality parameters of parts change obviously. The quality trend of machine learning training is better; the quality parameters of parts in traditional manufacturing process fluctuate greatly and decrease slowly with the time of feature recognition. In the test data, we randomly select 50 normal data and 50 abnormal data of an automobile product and compare the quality optimization in the model after training, respectively. We find that the efficiency of machine learning model diagnosis is relatively fast and can effectively solve the low efficiency problem caused by repetitive operation. Quality certification and optimization are the main channels to improve the economic changes of automobile market. In order to improve the market sales status, we also need to start from the manufacturing and reuse of parts. For some products that fail to meet the quality inspection standards, the reuse transformation can improve the utility rate of the products 


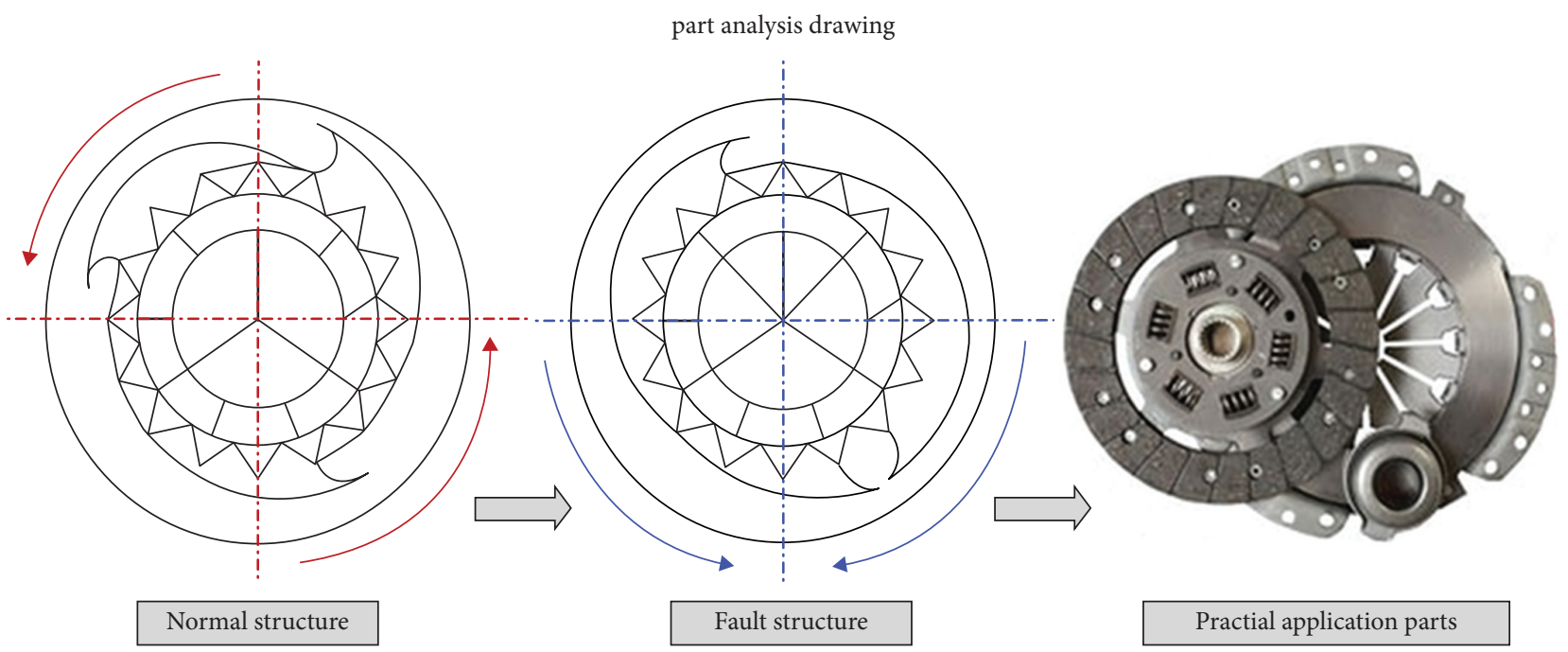

Figure 6: Actual application drawing after internal structure comparison of parts.

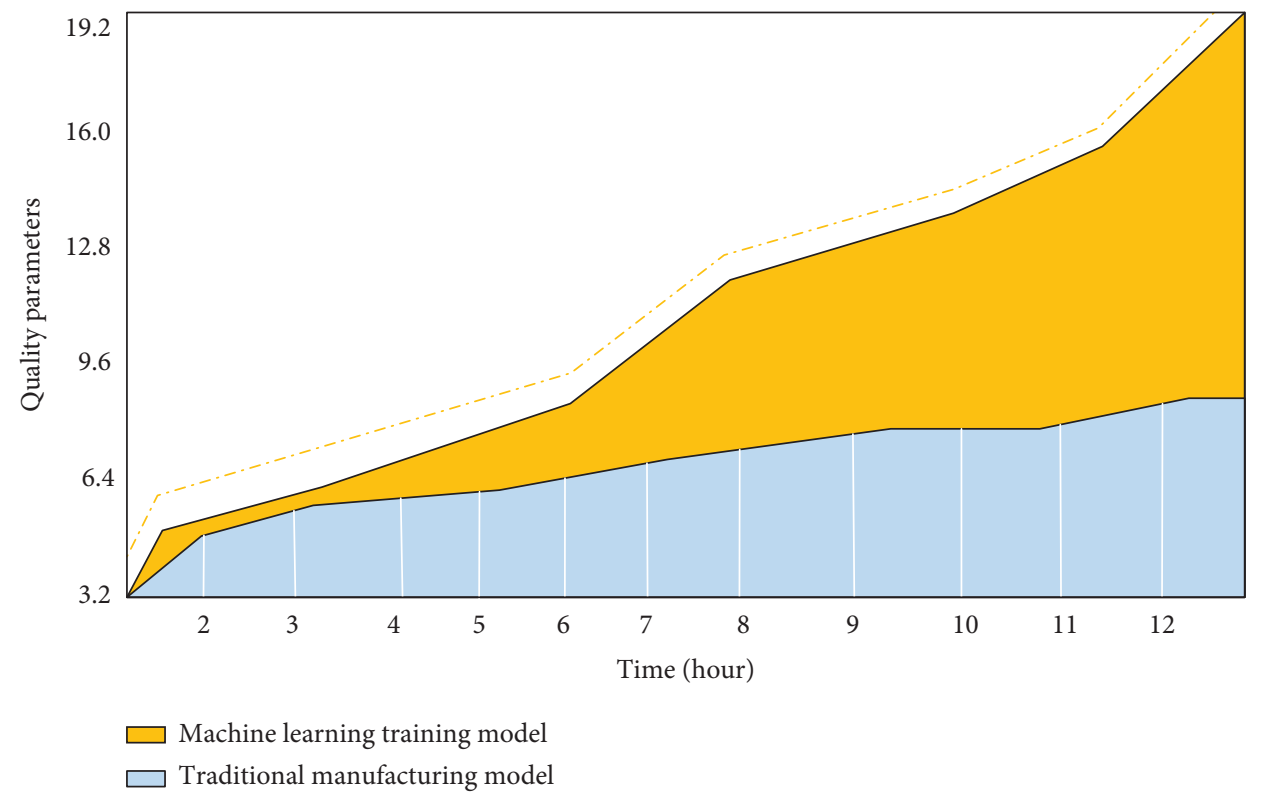

Figure 7: Changes of parts quality parameters before and after machine learning model training.

and reduce the average cost of the automobile industry to a certain extent. At the same time, in view of the uneven product quality level in the auto parts market, it is also necessary to start with the quality standard certification and use the quality certification mark to shift the manufacturing of parts from quantity to quality.

\section{Conclusion}

With the rise of national economic strength, the automobile industry is developing more and more rapidly. The increase of automobile types has brought new challenges to the manufacturing of parts and components. In the process of parts manufacturing, the selection of materials, process flow, processing, and testing technology are the main factors affecting product quality. This demand for higher quality puts forward requirements for the parts industry from the aspects of structural design, manufacturing mode, and quality inspection. In order to unify product quality standards and transform parts manufacturing from high quantity to high quality, we study the automobile parts manufacturing industry under the machine learning model. Firstly, starting from the market economy of automobile products, this paper explores the impact of cost price and material selection on automobile performance. It is found that the selection of raw materials has an obvious influence on the composition and quality of parts. Starting from the quality control management of parts, improve the core structure in the manufacturing process. The machine learning model is introduced to statistically analyze the material data. Using training samples to obtain the optimal results of quality data, it is added to the manufacturing 
process of auto parts. Finally, starting from the actual situation of China's automobile industry, the fault data are extracted from the parts process, and the simulation environment is used to optimize the quality parameters. In order to improve the manufacturing quality of parts, the fault analysis performance in sample making is trained by machine learning model. 3D printing technology is used to solve the problem of parts with high-precision requirements. This paper puts forward new measures and ideas for the reuse of parts and components in China. The results show that the auto parts manufacturing industry needs to change from paying attention to product quantity to paying attention to high-quality development. The product quality optimization based on machine learning model can improve the nonstandard operation in the manufacturing process of parts industry and provide new help for China's automobile industry in the market competition.

\section{Data Availability}

The datasets used during the current study are available from the corresponding author on reasonable request.

\section{Conflicts of Interest}

The authors declare that they have no conflicts of interest.

\section{Acknowledgments}

This work was sponsored in part by the "2021 Anhui University Humanities and Social Science Research Project (Grant no. SK2021A0479)" and "The sixth 2021 of Applied Social Science Research in Chuzhou (Grant no. A2021014).”

\section{References}

[1] m. Chen, "Research on quality control measures of automobile parts manufacturing," Metallurgy and materials, vol. 41 , no. 5 , pp. 17-18, 2021.

[2] K. Chen, "Promote enterprise quality management system certification and standardize the development of China's auto parts remanufacturing industry," Automobile and accessories, no. 19 , pp. 52-53, 2021.

[3] D. Wu, "Nondestructive testing technology and its application in automobile parts manufacturing process," Modern manufacturing technology and equipment, vol. 57, no. 9, pp. 149-150, 2021.

[4] Y. Lu, "Suggestions on optimization of internal control system of auto parts manufacturing enterprises," China foreign capital, no. 16, pp. 68-70, 2021.

[5] H. Jin and Y. Jiang, "Discussion on key technologies of remanufacturing internal combustion engine and its parts," Internal combustion engines and accessories, no. 21, pp. 192-193, 2021.

[6] N. Kong and P. Cui, "On the casting process of new die casting parts of automobile," Internal combustion engines and accessories, no. 13, pp. 105-106, 2021.

[7] C. Hu and Y. Wang, "Analysis of quality control and optimization measures for automobile and parts manufacturing," Times automobile, no. 13, pp. 153-154, 2021.

[8] S. Qiu, H. Zhao, and K. Fang, "Application of 3D printing and CNC processing in different stages of automobile and parts manufacturing," Automotive technologist, no. 6, pp. 33-37, 2021.

[9] J. Ge, P. Guo, and Q. Li, "Research on the development trend of automobile parts manufacturing technology," Auto parts, vol. 1, no. 5, pp. 107-109, 2021.

[10] C. Wang, "Analysis on key technologies of remanufacturing internal combustion engine and its parts," Internal combustion engines and accessories, vol. 5, no. 18, pp. 132-133, 2021.

[11] S. Zhang, X. Huang, Y. Chang, L. Yan, and W. Cheng, "Research progress and development trend of quantum machine learning in big data environment," Journal of University of Electronic Science and technology, vol. 50, no. 6, pp. 802-819, 2021.

[12] Q. Liu, j. Wang, J. Yin, Y. Chen, and J. Liu, "Application of anti machine learning in network intrusion detection," Journal of communications, vol. 42, no. 11, pp. 1-12, 2021.

[13] Z. Wang and L. Ge, "Credit risk quantification and decision analysis based on machine learning," Software engineering, vol. 24, no. 12, pp. 40-44, 2021.

[14] H. Liu, S. Gu, Q. Liu et al., "Alignment method of pipeline internal and external inspection data based on machine learning algorithm," Oil and gas storage and transportation, vol. 40, no. 11, pp. 1236-1241, 2021.

[15] H. Yu, W. Cheng, Z. Dai, J. Wang, and y. Xu, "Design of emergency material demand forecasting model based on machine learning," Electronic design engineering, vol. 29, no. 22, pp. 19-23, 2021. 